\title{
NEW EXACT SOLUTIONS TO THE MODIFIED FORNBERG-WHITHAM EQUATION
}

\author{
Ameina S. Nuseir
}

\begin{abstract}
In this paper a unified approach for finding soliton solutions is applied to the modified Fornberg-Whitham equation. Variety of peakon, periodic, and solitary new exact solutions are constructed.
\end{abstract}

\section{INTRODUCTION}

The study of nonlinear wave equations and their solutions is of great importance in many areas of physics. Travelling wave solutions are among the interesting types of solutions for the nonlinear partial differential equations. On the other hand, many nonlinear partial differential equations have been found to have a variety of travelling wave solutions. An example, is the well-known Korteweg-de Vries equation

$$
u_{\mathrm{t}}+6 u u_{x}+u u_{x x x}=0
$$

which is a mathematical model of waves on shallow water surfaces that has smooth solitary wave solutions [8]. Also, the Camassa-Holm equation

$$
u_{t}-u_{x x t}+3 u u_{x}=2 u_{x} u_{x x}+u u_{x x x}
$$

proposed by Camassa and Holm (1993)[1] is a model equation for the unidirectional nonlinear dispersive waves in shallow water. Due to its interesting mathematical properties, this equation has gained a lot of interest over the past decade. This equation has been found to have peakons, stumpons, cuspons, and composite wave solutions [9]. Nevertheless, it also has compactons [11]. Liu and coworkers found a new type of travelling wave solutions for the Camassa-Holm equation [12], which are defined on some semifinal bounded domains that posses properties of kink or antikink waves

Received February 23, 2012, accepted March 19, 2012.

Communicated by Yosikazu Giga.

2010 Mathematics Subject Classification: 35-04, 35Cxx, 35Dxx, 35Qxx.

Key words and phrases: Modified Fornberg-Whitham equation, Soliton solution, Exact solution in explicit form, Nonlinear partial differential equation. 
which they called kink-like waves and antikink-like waves. Later on, it has been found that the $\mathrm{CH}-\gamma$ equation

$$
u_{t}+c_{0} u_{x}+3 u u_{x}-\alpha^{2}\left(u_{x x t}+u u_{x x x}+3 u_{x} u_{x x}\right)+\gamma u_{x x x}=0
$$

posses kink-like waves when $\alpha \neq 0[4,15]$. Furthermore, the Fornberg-Whitham equation which is given as

$$
u_{t}-u_{x x t}+u_{x}=u u_{x x x}-u u_{x}+3 u_{x} u_{x x}
$$

was used to study the qualitative behavior of wave-breaking [8,9]. It admits a wave of greatest height, as a peaked limiting form of the travelling wave solution

$$
u(x, t)=A \exp \left(\frac{1}{2}\left|x-\frac{4}{3} t\right|\right),
$$

where $A$ is an arbitrary constant [3].

In 2006, Wazwaz [17] suggested studying the modified forms of the Camassa-Holm and the Degasperis-Procesi equations

$$
u_{t}-u_{x x t}+3 u^{2} u_{x}=2 u_{x} u_{x x}+u u_{x x x} ;
$$

and

$$
u_{t}-u_{x x t}+4 u^{2} u_{x}=3 u_{x} u_{x x}+u u_{x x x} .
$$

Then, many travelling wave solutions of Eqs. 5 and 6 have been obtained using tanh method, sine-cosine method and extended tanh method [17,18]. Using the bifurcation method of planar systems and numerical simulation of differential equations, Liu and Ouyang [13] obtained some peakon and solitary wave solutions. Moreover, Wang and Tang [16] obtained some new peakon and solitary wave solutions through some special phase orbits. Another study by Rui et al. [14] obtained some exact travelling wave solutions using the integral bifurcation method. Using the Homotopy perturbation method, Zhang et al. [21] solved Eqs. 5 and 6 to obtain some exact solutions.

He [5] used the bifurcation theory and the method of phase portraits analysis $[6,7,10]$ to investigate the modified Fornberg-Whitham $(\mathrm{mFW})$ equation:

$$
u_{t}-u_{x x t}+u_{x}=u u_{x x x}-u^{2} u_{x}+3 u_{x} u_{x x},
$$

and obtained some explicit peakon and solitary wave solutions.

In this article, a unified approach to find more explicit solutions for the $\mathrm{mFW}$ equation is used. 


\section{The Unified Approach for Finding Soliton Solutions}

Based on the randomness of the Painlevé analysis manifold, E. Fan [2] developed a method to find a rich variety of exact travelling wave solutions of a nonlinear evolution equation. At the same time, G. Xu and coworkers [20] published another article describing the same method and applied it to some nonlinear PDE's. In this approach, they considered nonlinear the evolution equations (NEE's) in two variables,

$$
F\left(u ; u_{t} ; u_{x} ; u_{x t} ; u_{x x} ; \ldots\right)=0
$$

where the subscripts denote partial derivatives, and $F$ is a polynomial in unknown function $u(x, t)$ and its derivatives. The travelling wave solutions to 8 can be written as

$$
u(x ; t)=u(\xi) ; \xi=x-c t
$$

in which $k$ is the wave number, and $c$ is the wave speed to be determined. Using the transformation in 9, Eq. 8 can be transformed to an ordinary differential equation of the type:

$$
G\left(u ; u^{\prime} ; u^{\prime \prime} ; \ldots\right)=0
$$

where $u^{\prime}=\frac{d u}{d \xi}$.

To introduce the concept of "rank", the term in the reduced ordinary differential equations will be written as:

$$
u^{k_{0}}\left(u^{\prime}\right)^{k_{1}}\left(u^{\prime \prime}\right)^{k_{2}} \ldots \ldots\left(u^{(m)}\right)^{k_{m}}
$$

where $k j(j=0 ; \ldots \ldots ; m)$ are real constants. Thus the rank of this term is defined as the number:

$$
0 \cdot k_{0}+1 \cdot k_{1}+2 \cdot k_{2}+\ldots \ldots \ldots+m \cdot k_{m} .
$$

Considering that the travelling wave solutions of many NEEs derived from soliton theory can be expressed as polynomials of special functions such as sech, tanh, sin, cos and the like. Now, if the rank of every term in 10 is even or odd, then the following truncated expansion

$$
u(\xi)=\sum_{j=0}^{m} a_{j} \phi^{j},
$$

can be taken, and the expansion variable $\phi=\phi(\xi)$ satisfies

$$
\left(\phi^{\prime}\right)^{2}=c_{0}+c_{1} \phi+c_{2} \phi^{2}+\ldots+c_{r} \phi^{r},
$$


where $m$ and $r$ are positive integers, and $\mathrm{a}_{\mathrm{j}}(\mathrm{j}=0,1, \ldots, \mathrm{m}), \mathrm{c}_{\mathrm{i}}(\mathrm{i}=0, \ldots, \mathrm{r})$ are constants the to be determined. To find the travelling wave solutions of Eq. 8, the following steps will be followed:

Step 1. To determine $m$ and $r$, one may substitute 11 into Eq. 10 and balance the highest derivative terms with the most nonlinear term in Eq.10. By doing so, a relation for $m$ and $r$ will be obtained. From this relation different possible values of $m$ and $r$ can be found.

Step 2. Substitute the series 11 along with 12 into Eq. 10; to get $P(\phi)=0$, where $P(\phi)$ is a polynomial in $\phi$. By equating the coefficients of each power of $\phi$ in $P(\phi)$ to zero, an algebraic system involving $a_{j}(j=0, \ldots, m), c_{i}(i=0, \ldots, r)$ and $c$ is obtained.

Step 3. By solving Eq. 12 and then substituting the solution in Eq. 8, some kinds of interesting travelling wave solutions will be obtained.

\section{Solving the Modified Fornberg-Whitham Equation}

To apply this method, the modified Fornberg-Whitham equation

$$
u_{t}-u_{x x t}+u_{x}=u u_{x x x}-u^{2} u_{x}+3 u_{x} u_{x x},
$$

should be transformed into an ODE. Let $\xi=x-c t$ and $u(x ; t)=u(\xi)$, then the equation will transform into

$$
c u^{\prime \prime \prime}-u u^{\prime \prime \prime}-3 u^{\prime} u^{\prime \prime}+u^{2} u^{\prime}+(1-c) u^{\prime}=0 .
$$

The ranks of the terms in Eq. 14 are 3,3,3,1 and 1 respectively. Therefore, we can use the above method.

First, find all derivatives of $u$ of orders 1,2 and 3 in terms of $\phi$

$$
u^{\prime}=a_{1} \phi^{\prime}+2 a_{2} \phi \phi^{\prime}+\ldots \ldots+m a_{m} \phi^{m-1} \phi^{\prime}=\phi^{\prime}\left(a_{1}+2 a_{2} \phi+\ldots \ldots+m a_{m} \phi^{m-1}\right),
$$

and

$$
u^{\prime \prime}=a_{1} \phi^{\prime \prime}+2 a_{2}\left(\phi \phi^{\prime \prime}+\phi^{\prime 2}\right)+\ldots \ldots+m a_{m}\left(\phi^{m-1} \phi^{\prime \prime}+(m-1) \phi^{m-2} \phi^{2}\right) .
$$

To find $\phi^{\prime \prime}$ we differentiate Eq. 12 and get

$$
\phi^{\prime \prime}=\frac{1}{2}\left(c_{1}+2 c_{2} \phi+\ldots \ldots+r c_{r} \phi^{r-1}\right) .
$$

Therefore,

$$
\begin{aligned}
u^{\prime \prime}= & \frac{1}{2}\left(c_{1}+2 c_{2} \phi+\ldots \ldots+r c_{r} \phi^{r-1}\right)\left(a_{1}+2 a_{2} \phi+\ldots \ldots+m a_{m} \phi^{m-1}\right) \\
& +\left(2 a_{2}+\ldots \ldots+m(m-1) a_{m} \phi^{m-2}\right)\left(c_{0}+c_{1} \phi+c_{2} \phi^{2}+\ldots+c_{r} \phi^{r}\right)
\end{aligned}
$$


and

$$
\begin{aligned}
& u^{\prime \prime \prime}=\frac{1}{2} \phi^{\prime} \\
& {\left[\begin{array}{l}
\left(2 c_{2}+6 c_{3} \phi+\cdots+r(r-1) c_{r} \phi^{r-2}\right)\left(a_{1}+2 a_{2} \phi+\cdots+m a_{m} \phi^{m-1}\right) \\
+\left(c_{1}+2 c_{2} \phi+\cdots+r c_{r} \phi^{r-1}\right)\left(6 a_{2}+18 a_{3} \phi+\cdots+3 m(m-1) a_{m} \phi^{m-2}\right) \\
+2\left(c_{0}+c_{1} \phi+c_{2} \phi^{2}+\cdots+c_{r} \phi^{r}\right)\left(6 a_{3}+\cdots+m(m-1)(m-2) a_{m} \phi^{m-3}\right)
\end{array}\right]}
\end{aligned}
$$

Substitute Eqs. 15, 16, 18, and 19 into Eq. 14 and take $\phi^{\prime}$ as common factor, then the highest nonlinear terms in $u u^{\prime \prime \prime}$ is $\phi^{2 m+r-3}$; while that for the term $u^{2} u^{\prime}$ is $\phi^{3 m-1}$.

Now, if we balance the powers, we obtain the relation $r=m+2$ between $m$ and $r$. If we take $m=1$, then $r=3$, so

$$
u(\xi)=a_{0}+a_{1} \phi(\xi)
$$

and

$$
\left(\phi^{\prime}\right)^{2}=c_{0}+c_{1} \phi+c_{2} \phi^{2}+c_{3} \phi^{3}
$$

The solutions for Eq. 20 are:

$$
\begin{aligned}
& \phi=\frac{-c_{2}}{c_{3}} \operatorname{Sec}^{2}\left(\frac{\sqrt{-c_{2}}}{2} \xi\right), \text { if } c_{0}=c_{1}=0, c_{2}<0, \text { and } \\
& \phi=\frac{-c_{2}}{c_{3}} \operatorname{Sech}^{2}\left(\frac{\sqrt{c_{2}}}{2} \xi\right), \text { if } c_{0}=c_{1}=0, c_{2}>0 .
\end{aligned}
$$

So the following two solutions for $u(x, t)$ will be obtained:

$$
\begin{aligned}
u= & \frac{1}{4}\left(10 c_{2}-2\left(4+\sqrt{3} \sqrt{4-5 c_{2}^{2}}\right)\right) \\
& -\frac{15}{2} c_{2} \operatorname{Sec}\left(\frac{1}{2} \sqrt{-c_{2}}\left(-2\left(4+\sqrt{3} \sqrt{4-5 c_{2}^{2}}\right) t+x\right)\right)^{2}, c_{2}>0
\end{aligned}
$$

and

$$
\begin{aligned}
u= & \frac{1}{4}\left(10 c_{2}-2\left(4+\sqrt{3} \sqrt{4-5 c_{2}^{2}}\right)\right) \\
& -\frac{15}{2} c_{2} \operatorname{Sech}\left(\frac{1}{2} \sqrt{-c_{2}}\left(-2\left(4+\sqrt{3} \sqrt{4-5 c_{2}^{2}}\right) t+x\right)\right)^{2}, c_{2}<0
\end{aligned}
$$

Now take $m=2$, then $r=4$, and 


$$
\begin{aligned}
u(\xi) & =a_{0}+a_{1} \phi(\xi)+a_{2} \phi^{2}(\xi), \\
\left(\phi^{\prime}\right)^{2} & =c_{0}+c_{1} \phi+c_{2} \phi^{2}+c_{3} \phi^{3}+c_{4} \phi^{4} .
\end{aligned}
$$

Then solve Eq. 21 and substitute the solutions for $u(\xi)$ into Eq. 14. The following solutions will be found:

If $c_{0}=c_{1}=c_{2}=c_{3}=0, c_{4}>0$, then $\phi=-\frac{1}{\sqrt{c_{4}} \xi}$, and $u_{1}=-2-\sqrt{3}+\frac{30}{(x-c t)^{2}}$, where $c=4(2 \mp \sqrt{3})$.

If $c_{0}=c_{1}=c_{3}=0, c_{2}<0, c_{4}>0$, then $\phi=\sqrt{\frac{-c_{2}}{c_{4}}} \operatorname{Sec}\left(\sqrt{-c_{2}} \xi\right)$, and $u_{2}=\frac{1}{4}\left(40 c_{2}-c\right)-30 c_{2} \operatorname{Sec}^{2}\left(\sqrt{-c_{2}}(x-c t)\right)$, where $c=4\left(2 \mp \sqrt{3} \sqrt{1-20 c_{2}^{2}}\right)$.

If $c_{1}=c_{3}=0, c_{0}=\frac{c_{2}^{2}}{4 c_{4}}, c_{2}>0, c_{4}>0$, then $\phi=\sqrt{\frac{c_{2}}{2 c_{4}}} \operatorname{Tan}\left(\sqrt{\frac{c_{2}}{2}} \xi\right)$, and

$u_{3}=\frac{1}{4}\left(40 c_{2}-c\right)+15 c_{2} \operatorname{Tan}^{2}\left(\frac{\sqrt{c_{2}}(x-c t)}{\sqrt{2}}\right)$, where $c=4\left(2 \mp \sqrt{3} \sqrt{1-5 c_{2}^{2}}\right)$.

If $c_{0}=c_{1}=c_{3}=0, c_{2}>0, c_{4}<0$, then $\phi=\sqrt{\frac{-c_{2}}{c_{4}}} \operatorname{Sech}\left(\sqrt{c_{2}} \xi\right)$ and $u_{4}=\frac{1}{4}\left(40 c_{2}-c\right)-30 c_{2} \operatorname{Sech}^{2}\left(\sqrt{c_{2}}(x-c t)\right)$, where $c=4\left(2 \mp \sqrt{3} \sqrt{1-20 c_{2}^{2}}\right)$.

If $c_{1}=c_{3}=0, c_{0}=\frac{c_{2}^{2}}{4 c_{4}}, c_{2}<0, c_{4}>0$, then $\phi=\sqrt{\frac{-c_{2}}{2 c_{4}}} \operatorname{Tanh}\left(\sqrt{\frac{-c_{2}}{2}} \xi\right)$, and $u_{5}=\frac{1}{4}\left(40 c_{2}-c\right)-15 c_{2} \operatorname{Tanh}^{2}\left(\frac{\sqrt{-c_{2}}(x-c t)}{\sqrt{2}}\right)$, where $c=4\left(2 \mp \sqrt{3} \sqrt{1-5 c_{2}^{2}}\right)$.

If $c_{0}=c_{1}=0, c_{2}<0$, then $\phi=-\frac{c_{2} \operatorname{Sec}^{2}\left[\frac{\sqrt{-c_{2}}}{2} \xi\right]}{2 \sqrt{-c_{2} c_{4}} \operatorname{Tan}\left[\frac{\sqrt{-c_{2}}}{2} \xi\right]+c_{3}}$, and

$$
u_{6}=\left[\begin{array}{l}
\frac{20 c_{2} c_{4}-2 c c_{4}}{8 c_{4}}+\frac{30 c_{2}^{2} c_{4} \operatorname{Sec}^{4}\left[\frac{\sqrt{-c_{2}}(x-c t)}{2}\right]}{\left(2 \sqrt{c_{2} c_{4}}+2 \sqrt{-c_{2} c_{4}} \operatorname{Tan}\left[\frac{\sqrt{-c_{2}}(x-c t)}{2}\right]\right)^{2}} \\
-\frac{30 c_{2}^{\frac{3}{2}} \sqrt{c_{4}} \operatorname{Sec}^{2}\left[\frac{\sqrt{-c_{2}}(x-c t)}{2}\right]}{2 \sqrt{c_{2} c_{4}}+2 \sqrt{-c_{2} c_{4}} \operatorname{Tan}\left[\frac{\sqrt{-c_{2}}(x-c t)}{2}\right]}
\end{array}\right],
$$

where, $c=2\left(4 \mp \sqrt{3} \sqrt{4-5 c_{2}^{2}}\right)$.

If $c_{0}=c_{1}=0, c_{2}>0$, then $\phi=\frac{c_{2} \operatorname{Sech}^{2}\left[\frac{\sqrt{c_{2}}}{2} \xi\right]}{2 \sqrt{c_{2} c_{4}} \operatorname{Tanh}\left[\frac{\sqrt{c_{2}}}{2} \xi\right]-c_{3}}$, and

$$
u_{7}=\left[\begin{array}{l}
\frac{20 c_{2} c_{4}-2 c c_{4}}{8 c_{4}}+\frac{30 c_{2}^{2} c_{4} \operatorname{Sech}^{4}\left[\frac{\sqrt{c_{2}}(x-c t)}{2}\right]}{\left(-2 \sqrt{c_{2} c_{4}}+2 \sqrt{c_{2} c_{4}} \operatorname{Tanh}\left[\frac{\sqrt{c_{2}}(x-c t)}{2}\right]\right)^{2}} \\
+\frac{30 c_{2}^{\frac{3}{2}} \sqrt{c_{4}} \operatorname{Sech}^{2}\left[\frac{\sqrt{c_{2}}(x-c t)}{2}\right]}{\left.-2 \sqrt{c_{2} c_{4}}+2 \sqrt{c_{2} c_{4}} \operatorname{Tanh}^{2} \frac{\sqrt{c_{2}}(x-c t)}{2}\right]}
\end{array}\right],
$$


where $c=2\left(4 \mp \sqrt{3} \sqrt{4-5 c_{2}^{2}}\right)$.

The following figures display different type of solutions.

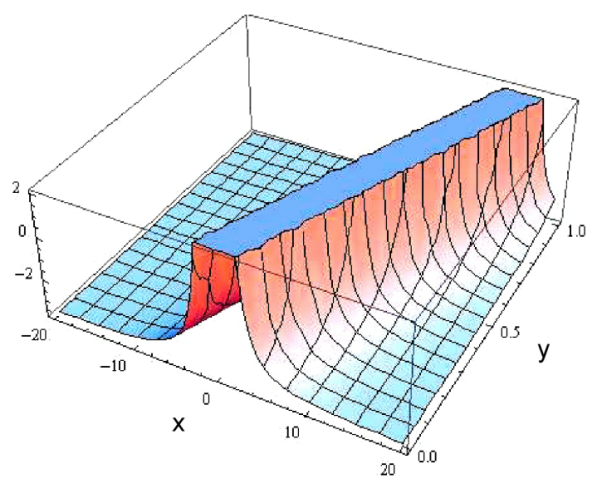

Fig. 1. The peakon solution $u_{1}=-2-\sqrt{3}+\frac{30}{(x-c t)^{2}}$.

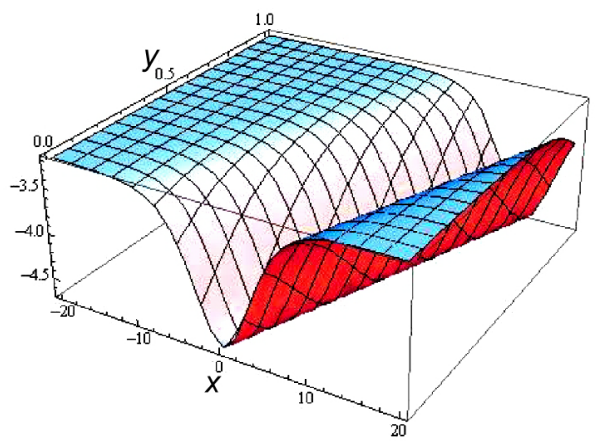

Fig. 2. The soliton solution $u_{7}$ when $c_{2}=1 / 5$, and $c_{4}=1$.

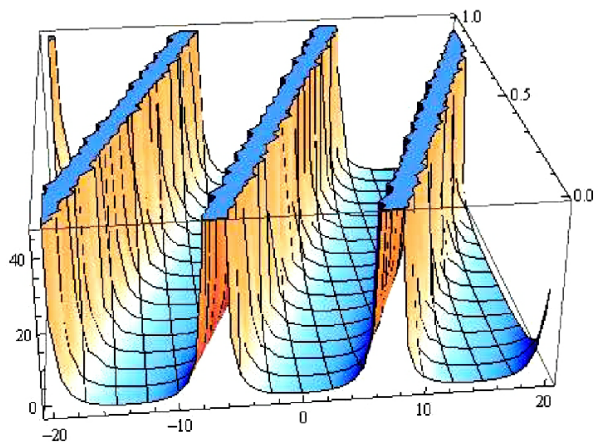

Fig. 3. The periodic solution $u_{6}$ when $c_{2}=-1 / 5$, and $c_{4}=1$. 


\section{CONCLUSION}

As seen from above, applying this method we've got peakon, periodic and solitary wave solutions to the modified Fornberg-Whitham equation that are not found by the bifurcation theory and the method of phase portraits analysis. So this method is very useful to construct several kinds of exact solutions for nonlinear partial differential equations.

\section{REFERENCES}

1. R. Camassa and D. Holm, An integrable shallow wave equation with peaked solitons, Phys. Rev. Lett., 71 (1993), 1661-1664.

2. E. Fan, Uniformly constructing a series of explicit exact solutions to nonlinear equations in mathematical physics, Chaos Solitons and Fractals, 16 (2003), 819-839.

3. B. Fornberg and G. B. Whitham, A numerical and theoretical study of certain nonlinear wave phenomena, Philos. Trans. Roy. Soc. Lond. Ser. A., 289 (1978), 373-404.

4. B. Guo and Z. Liu, Two new types of bounded waves of CH-c equation, Sci. China, Ser. A., 48 (2005), 1618-1630.

5. B. He, Q. Meng and S. Li, Explicit peakon and solitary wave solutions for the modified Fornberg-Whitham equation, Appl. Math. Comp., 217 (2010), 1976-1982

6. B. He, J. B. Li, Y. Long and W. G. Rui, Bifurcations of travelling wave solutions for a variant of Camassa-Holm equation, Nonlinear Analysis RWA, 9 (2008), 222-232.

7. B. He, Y. Long and W. G. Rui, New exact bounded travelling wave solutions for the Zhiber-Shabat equation, Nonlinear Analysis TMA, 71 (2009), 636-1648.

8. J. Lenells, Traveling wave solutions of the Camassa-Holm and Korteweg-de Vries equations, J. Nonlinear Math. Phys., 11 (2004), 508-520.

9. J. Lenells, Traveling wave solutions of the Camassa-Holm equation, J. Diff. Eq., 217 (2005), 393-430.

10. J. B. Li and Z. R. Liu, Smooth and non-smooth traveling waves in a nonlinearly dispersive equation, Appl. Math. Model., 25 (2000), 41-56.

11. Z. Liu and C. Chen, Compactons in a general compressible hyperelastic rod, Chaos, Soliton Fract., 22 (2004), 627-640.

12. Z. Liu, Q. Li and Q. Lin, New bounded traveling wave solutions of Camassa-Holm equation, Int. J. Bifurcation Chaos, 14 (2004), 3541-3556.

13. Z. R. Liu and Z. Y. Ouyang, A note on solitary waves for modified forms of CamassaHolm and Degasperis-Procesi equations, Phys. Lett. A, 366 (2007), 377-381.

14. W. G. Rui, B. He, S. L. Xie and Y. Long, Application of the integral bifurcation method for solving modified Camassa-Holm and Degasperis-Procesi equations, Nonlinear Analysis TMA, 71 (2009), 3459-3470. 
15. M. Tang and W. Zhang, Four types of bounded wave solutions of CH-c equation, Sci. China, Ser. A, 50 (2007), 132-152.

16. Q. D. Wang and M. Y. Tang, New exact solutions for two nonlinear equations, Phys. Lett. A, 372 (2008), 2995-3000.

17. A. M. Wazwaz, Solitary wave solutions for modified forms of Degasperis-Procesi and Camassa-Holm equations, Phys. Lett. A, 352 (2006), 500-504.

18. A. M. Wazwaz, New solitary wave solutions to the modified forms of Degasperis-Procesi and Camassa-Holm equations, Appl. Math. Comput., 186 (2007), 130-141.

19. G. B. Whitham, Variational methods and applications to water wave, Proc. R. Soc. Lond. A, 299 (1967), 6-25.

20. G. Xu, Z. Li and Y. Liu, Exact solutions to a large class of nonlinear evolution equations, Chinese J. Phys., 41 (2003), 232-241.

21. B. G. Zhang, S. Y. Li and Z. R. Liu, Homotopy perturbation method for modified Camassa-Holm and Degasperis-Procesi equations, Phys. Lett. A, 372 (2008), 18671872.

Ameina S. Nuseir

Department of Mathematics and Statistics

Jordan University of Science and Technology

P. O. Box 3030

Irbid 22110

Jordan

E-mail: anuseir@just.edu.jo 